Research Paper

\title{
Parity Correlates with the Timing of Developing Endometrial Cancer, But Not Subtype of Endometrial Cancer
}

\author{
Qi Chen ${ }^{1,2}$, Mancy Tong2 2 Fang Guo 1 , Sandy Lau ${ }^{2}$, Min Zhao ${ }^{\bowtie}$ \\ 1. The Hospital of Obstetrics \& Gynaecology, Fudan University, China \\ 2. Department of Obstetrics \& Gynaecology, The University of Auckland, New Zealand \\ 3. Auckland Bioengineering Institute, The University of Auckland, New Zealand \\ 4. Wuxi Maternity and Children Hospital, Nanjing Medical University, China
}

$\triangle$ Corresponding author: Dr. M Zhao, 48 Huaishu Street, Wuxi, China, Wuxi Maternity and Children Hospital, Nanjing Medical University, China, Email: q.chen@auckland.ac.nz, Phone: 86-13611691734

(C) 2015 Ivyspring International Publisher. Reproduction is permitted for personal, noncommercial use, provided that the article is in whole, unmodified, and properly cited. See http://ivyspring.com/terms for terms and conditions.

Received: 2015.05.18; Accepted: 2015.07.27; Published: 2015.09.03

\begin{abstract}
Parity is one of well-known risk factors of endometrial cancer, but it is less clear whether parity is associated with the timing of developing endometrial cancer, and with the incidence of subtype of endometrial cancer. Data on 902 patients including age at diagnosis, age at menarche and menopause, parity and age at last birth from two obstetrics and gynaecology hospitals in China was analysed. $5.6 \%$ patients were nulliparous, $53.4 \%$ patients had one live birth, $27.7 \%$ patients had two live births and $13.3 \%$ patients had three or more live births. Patients at diagnosis who had three or more live births were significantly older than patients who were nulliparous or had one or two live births with the median age at diagnosis of 63 versus 51 or 52 or 59 years respectively. The percentage of patients with three or more live births at diagnosis before menopause was significantly lower than patients who were nulliparous, had one live birth or had two live births (10\% versus

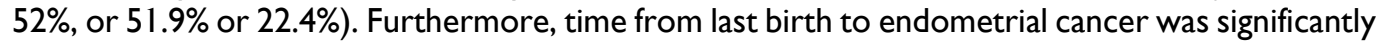
longer in patients who had three or more live births than patients who had two live births or had one live birth (35 versus 31, or 24 years). However, there was no difference in the incidence of subtypes of endometrial cancer according to parity. Our data suggests parity is negatively correlated with the time onset of endometrial cancer and not associated with the incidence of endometrial cancer subtypes.
\end{abstract}

Key words: parity, endometrial cancer, time onset, type I and type II, menopause

\section{Introduction}

Endometrial cancer is the most common cancer of the female reproductive tract in developed countries and is the third most common cause of death from female cancers. It occurred in 320,000 women and caused 76,000 deaths globally in 2012 [1]. The exact cause of endometrial cancer remains unknown, although several risk factors have been identified. These risk factors include nulliparity, late menopausal age (after 50 years old), diabetes mellitus, hypertension, obesity, BMI, early menarche, ethnicity and family history of endometrial cancer [2-7].
Unopposed estrogen exposure to endometrium is a common cause of endometrial cancer [8]. Pregnancy results in large changes of hormone levels and balance. During pregnancy, increased levels of progesterone change the hormonal balance which shifts toward more progesterone to oppose estrogen levels. For this reason, parous women have a lower risk of developing endometrial cancer compared to nulliparous women because of the reduced levels of estrogen $[4,7]$. Increased number of births has a protective effect on the development of endometrial cancer $[9,10]$. 
Other studies have shown that age at last birth also lowers the risk factor for developing endometrial cancer [2]. A number of studies also investigated the association of risk factors and endometrial cancer in postmenopausal women [11], but the hormone levels in women are different prior to and after menopause. Taken together, the incidence of endometrial cancer is associated with parity and age at last birth. However reports studying whether parity and time after last birth are associated with the time of onset for developing endometrial cancer, and the age at diagnosis are limited.

Endometrial cancer is commonly divided into type I and type II, based on clinical features and pathogenesis. The two subtypes are genetically distinct [12]. Type I endometrial cancer is estrogen dependent and represent $75-90 \%$ of endometrial cancer[12], while type II is estrogen independent which is not associated with increased exposure to estrogen. Whether parity is associated with the incidence of type I or type II endometrial cancer has not been fully investigated. In this study we performed a retrospective analysis of data obtained from two university teaching Obstetrics and Gynaecological hospitals serving diverse urban and rural areas in China to investigate the association between parity and time onset of endometrial cancer, and the incidence of subtypes of endometrial cancer in Chinese women.

\section{Materials and Methods}

\section{Study subjects}

This retrospective data was collected and pooled together from two Obstetrics \& Gynaecology university teaching hospitals which serve a diverse urban and rural population in China. In this study total 902 women with endometrial cancer were included. Of them, 493 patients were from The Hospital of Obstetrics and Gynaecology, Fudan University, which is the largest specialised Obstetrics and Gynaecology teaching hospital in the largest city in China with a population of 20 million from November 2009 to December 2011. 409 patients were from Wuxi Maternity and Children Hospital of Nanjing Medical University which is located in a city with a population of 6 million from January 2008 to May 2014. Data on all patients with a primary diagnosis of endometrial cancer administered to the two hospitals were collected according to the medical records of patients including clinical, intra-operation and pathological findings. Data included clinical characteristics of patients with endometrial cancer such as age at diagnosis, age at menarche and menopause which were self-reported, parity, age at last birth, hypertension, diabetes, and delivery mode. The Clinical characteristics of women with endometrial cancer were summarised in Table 1.

\section{Statistical analysis}

The statistical difference in age at diagnosis of endometrial cancer or the incidence of patients before menopause or the incidence of patients after menopause at diagnosis was assessed with Fisher's exact test using the Prism software package. The statistical difference in time from age at menopausal to endometrial cancer or time since last birth to endometrial cancer was assessed with Fisher's exact test using the Prism software package. The statistical difference in delivery mode among the subgroups according to parity was assessed with Fisher's exact test using the Prism software package. P-values of $<0.008$ were considered significant after the application of Bonferroni corrections.

\section{Results}

\section{Clinical characteristics of the study population}

The clinical characteristics of patients $(n=902)$ were summarised in Table 1 . The median age of patients at diagnosis was 55 (range 21-85) years old. Of 902 patients, 51(5.6\%) patients were nulliparous, 481 (53.4\%) patients had one live birth, 250 (27.7\%) patients had two live births and 120 (13.3\%) patients had three or more live births. Of 902 patients, 344 (38.1\%) patients were diagnosed before menopause and 558 $(61.9 \%)$ women were diagnosed after menopause. There were only $29(3.3 \%)$ patients who had menarche before 12 years old, $87(9.6 \%)$ patients with diabetes mellitus and 273 (30\%) patients with hypertension. Of 902 patients, $765(84.8 \%)$ patients were diagnosed with type I of endometrial cancer and 137 (15.2\%) patients were diagnosed with type II of endometrial cancer.

Table 1: Clinical characteristics of women with endometrial cancer

\begin{tabular}{ll}
\hline & $\mathrm{N}=902$ \\
\hline $\begin{array}{l}\text { Age at diagnosis (years old, median/range) } \\
\text { Parity (number, \%) }\end{array}$ & $55(21-85)$ \\
0 & $51(5.6 \%)$ \\
1 & $481(53.4 \%)$ \\
2 & $250(27.7 \%)$ \\
$\geq 3$ & $120(13.3 \%)$ \\
Menopause & \\
Perimenopause (number, \%) & $344(38.1 \%)$ \\
Postmenopause (number, \%) & $558(61.9 \%)$ \\
Age at menarche (years) (number, \%) & \\
$\leq 12$ years old & $29(3.3 \%)$ \\
$\geq 13$ years old & $873(96.7 \%)$ \\
Age at last birth (years old, median/range) & $27(19-51)$ \\
Type I & $765(84.8 \%)$ \\
Type II & $137(15.2 \%)$ \\
\hline
\end{tabular}




\section{Age at diagnosis of endometrial cancer was negatively correlated with parity}

To investigate the association between age at diagnosis and parity, we divided the study population into four subgroups based on parity of patients (Table 2). The median age at diagnosis in patients who had three or more live births was 63 years old, which was significantly older than patients who were nulliparous (51 years old), had one live birth (52 years old) or had two live births (59 years old). Similarly, the median age at diagnosis in patients who had two live births was significantly older than patients who had never been birth or patients who had one live birth. There was no statistical difference in median age at diagnosis between patients who had never been birth and patients who had one live birth $(p=0.08)$. In addition, the proportion of patients who were diagnosed before menopause was significantly higher in patients who were nulliparous $(51 \%)$ or had one live birth $(51.9 \%)$, compared to that in patients who had two live births $(22.4 \%)$ and those who had three or more live births $(10 \%)$ (Table 2, $\mathrm{p}=0.001$ ). Similarly, the proportion of patients who were diagnosed after menopause was significantly lower in patients who were nulliparous $(49 \%)$ or had one live birth $(48 \%)$, compared to that in patients who had two live births $(77.6 \%)$ and those who had three or more live births (90\%) (Table 2, $\mathrm{p}=0.001$ ).

\section{The time from last birth and menopause to endometrial cancer was negatively correlated with parity}

We then analysed the time since last birth to the diagnosis of endometrial cancer in three sub-groups according to parity (Table 3). The median time from last birth to endometrial cancer was 35 years in patients who had three or more live births, which was significantly longer compared to that in patients who had one live birth (24 years) and who had two live births (31 years) $(p=0.0004)$. Similarly, the time from last birth to endometrial cancer was significantly longer in patients who had two live births than that in patients who had one live birth $(p=0.0001)$. We also analysed the time from menopause to endometrial cancer for patients who were diagnosed after menopause (Table 3 ). The median years from menopause to endometrial cancer was 15 years in patients who had three or more live births, which was significantly longer than that in patients who had two live births (10 years) and patients who had one live birth (5 years). The median years from menopause to endometrial cancer were also significantly longer in patients who had two live births than that in patients who had one live birth $(\mathrm{p}=0.001)$.

\section{Parity was not correlated with the incidence of subtype of endometrial cancer}

We also analysed whether parity correlated with the incidence of subtype of endometrial cancer (Table 4). The majority of endometrial cancer was type I $(84.8 \%)$ in our study cohort and there was no statistical difference in the proportion of type I and type II endometrial cancer among four subgroups according to parity $(\mathrm{p}>0.05)$.

Table 2: The association between parity and the age of developing endometrial cancer

\begin{tabular}{lllll}
\hline & No child $(\mathrm{n}=51)$ & 1 child $(\mathrm{n}=481)$ & 2 children $(\mathrm{n}=250)$ & 3 more children $(\mathrm{n}=120)$ \\
\hline $\begin{array}{l}\text { Age at diagnosis (years old, me- } \\
\text { dian/ range) }\end{array}$ & $51(21-77)$ & $52(24-73)$ & $59(39-78)^{\mathrm{a}, \mathrm{b}}$ & $63(37-85)^{\mathrm{a}, \mathrm{b}, \mathrm{c}}$ \\
& & & \\
$\begin{array}{l}\text { Diagnosis before menopause } \\
\text { (number, \%, upper lower CL) }\end{array}$ & $26(51 \%)$ & $250(51.9 \%)$ & $56(22.4 \%)^{\mathrm{a}, \mathrm{b}}$ & $12(10 \%)^{\mathrm{a}, \mathrm{b}, \mathrm{c}}$ \\
& $(36.6 \%, 65.2 \%)$ & $(47.4 \%, 56.5 \%)$ & $(17.4 \%, 28.1 \%)$ & $(5.3 \%, 16.8 \%)$ \\
$\begin{array}{l}\text { Diagnosis after menopause } \\
\text { (number, \%, upper lower CL) }\end{array}$ & $25(49 \%)$ & $231(48.0 \%)$ & $194(77.6 \%)^{\mathrm{a}, \mathrm{b}}$ & $108(90 \%)^{\mathrm{a}, \mathrm{b}}$ \\
\end{tabular}

a: $p<0.001$ compared to no child; $b$ : $p<0.001$ compared to one child; c: $p<0.001$ compared to two children.

Table 3: The association between the time from last birth or menopause to endometrial cancer and parity

\begin{tabular}{llll}
\hline & No child $(\mathrm{n}=51)$ & 1 child $(\mathrm{n}=481)$ & 2 children $(\mathrm{n}=250)$ \\
\hline $\begin{array}{l}\text { Time from last birth to cancer } \\
\text { (years, median/range) }\end{array}$ & NA & $24(2-53)$ & $31(4-54)^{\mathrm{b}}$ \\
& & $5(0-23)$ & $35(7-57)^{\mathrm{b}, \mathrm{c}}$ \\
$\begin{array}{l}\text { Time from menopause to cancer } \\
\text { (years, median/range) }\end{array}$ & $7(0-25)$ & $10-30)^{\mathrm{b}}$ & $15(0-40)^{\mathrm{a}, \mathrm{b}, \mathrm{c}}$
\end{tabular}

a: $\mathrm{p}<0.001$ compared to no child; $\mathrm{b}$ : $\mathrm{p}<0.001$ compared to one child; : $\mathrm{p}<0.001$ compared to two children. 
Table 4: The association between parity and the subtype of endometrial cancer

\begin{tabular}{|c|c|c|c|c|}
\hline & No child $(n=51)$ & 1 child $(n=481)$ & 2 children $(n=250)$ & 3 more children $(n=120)$ \\
\hline Type I (number, \%, upper lower CL) & $\begin{array}{l}46(90.1 \%) \\
(78.6 \%, 96.7 \%)\end{array}$ & $\begin{array}{l}411(85.4 \%) \\
(81.9 \%, 88.5 \%)\end{array}$ & $\begin{array}{l}213(85.2 \%) \\
(80.2 \%, 89.4 \%)\end{array}$ & $\begin{array}{l}95(79.2 \%) \\
(70.8 \%, 86.1 \%)\end{array}$ \\
\hline Type II (number, \%, upper lower CL) & $\begin{array}{l}5(9.9 \%) \\
(3.3 \%, 21.4 \%)\end{array}$ & $\begin{array}{l}70(14.6 \%) \\
(11.5 \%, 18.1 \%)\end{array}$ & $\begin{array}{l}37(14.8 \%) \\
(10.6 \%, 19.8 \%)\end{array}$ & $\begin{array}{l}25(20.8 \%) \\
(13.9 \%, 29.2 \%)\end{array}$ \\
\hline
\end{tabular}

\section{Discussion}

The involvement of hormonal factor in developing endometrial cancer is well documented. Increase the levels of circulating estrogen increase the risk of developing endometrial cancer. In this retrospective study data collected from two obstetrics \& gynaecology hospitals, we have found that the median age of patients at diagnosis of endometrial cancer is younger in Chinese women compared to the findings in the literatures and negatively correlated with parity. In addition, we found that parity is not associated with the incidence of type I or type II endometrial cancer.

A number of studies reported that the incidence of endometrial cancer depends on the ethnicity and geographical area [13]. African American women have lower incidence of endometrial cancer than Caucasian women [6]. Ethnicity is also shown to be associated with endometrial cancer survival [14], and Asian women have had improved outcome of endometrial cancer compared to non-Asian women [15]. A recent study reported that the age at diagnosis of endometrial cancer in Asian women including Asian native and Asian immigrants, is younger than non-Asians [15]. Most patients with endometrial cancer were diagnosed between the ages of 60 and 70 years old, and the average age is 61 years old in developed countries [16]. In our current study, our data shows that the median age of patients at diagnosis was 55 years old in Chinese women, which is younger than other patients in developed countries [17]. Furthermore, our data also shows that $38.1 \%$ of patients were diagnosed before menopause which is higher than in developed countries (less than 25\%). We do not know the exact reason for such a difference, but studies suggested that hormone, cancer characteristics including histological subtype vary by ethnicities $[8$, $18,19]$.

The main risk factor for endometrial cancer is an excess of endogenous or exogenous estrogen without adequate opposition by progesterone. Study suggested that there is a positive correlation between higher doses and/or longer periods of unopposed estrogen and higher risk of endometrial cancer [20]. During pregnancy, the hormonal balance shifts toward more progesterone. Elevated progesterone may inhibit estrogen associated endometrial cell prolifera- tion and promote endometrial cell differentiation and apoptosis [21-23]. These studies suggest that having many pregnancies may protect against endometrial cancer compared with women who have never been pregnant. In our current study, our data shows that the $60 \%$ of patients with endometrial cancer were nulliparous or only had one live birth, while $27.7 \%$ of patients with endometrial cancer had two live births and $13.3 \%$ of patients with endometrial cancer had three more live births. Consistent with other studies, our result confirms that parity is strongly associated with a decreasing risk for the development of endometrial cancer. Another interesting finding in our current study is that the median age at diagnosis in patients who had never been pregnancy or patients who only had one live birth was 47.3 years old and 51.1 years old, respectively, while the median age at diagnosis in patients who had two live births or patients who had three or more live births was 58.5 years old or 63 years old, respectively. This was significantly older than patients who had never been pregnant or patients who only had one live birth. Furthermore, we also found that $51 \%$ of patients who have either had never been pregnant or had one live birth were diagnosed before menopause. However, only $22.4 \%$ of patients who had two live births and $10 \%$ of patients who had three or more live births were diagnosed before menopause, which is significant lower. Taken together, our result suggests that parity is negatively correlated with the age of patients at diagnosis of endometrial cancer.

A recent study suggested that age at last birth was independently associated with a lower risk of developing endometrial cancer [2]. In the current study, our data shows that the median age at last birth is 27 years old regardless parity which is consists with other studies' findings. We then questioned whether the time from last birth to endometrial cancer presentation is correlated with parity. Interestingly, we found that patients who had three or more live births had a significant longer period between time of last birth to endometrial cancer presentation compared to patients who had two live births or one live birth (34.7 years, versus 31.1 years or 23 years). We also analysed the time from menopause to endometrial cancer in patients who diagnosed with endometrial cancer after menopause. We found that the time from menopause to endometrial cancer in patients 
who had two or three or more live births was also significantly longer than that in patients who had one live birth (10 years or 15 years versus 5 years). Our data suggests that parity is positively correlated with the time from last birth to endometrial cancer presentation regardless of menopause.

Endometrial cancer is commonly divided into type I which is estrogen dependent and type II, which is estrogen independent. Whether parity correlated with the incidence of subtype of endometrial cancer has not been investigated. In our current study, our data shows that there was no statistical difference in the proportion of type I or type II endometrial cancer among the four subgroups according to parity, suggesting parity may not be associated with the incidence of subtype of endometrial cancer. This finding was surprising as the shift in progesterone/estrogen balance during pregnancy is thought to have a protective effect against the development endometrial cancer, yet type II of endometrial cancer is thought to be estrogen independent. However, a recent study reported that two types of endometrial cancer share many common risk factors for development of endometrial cancer and suggested that type II endometrial cancer may not be completely estrogen independent [24]. Further studies focusing on the association between the subtypes of endometrial cancer and parity are required to confirm our observation.

Having more menstrual cycles during a woman's lifetime, either from early menarche (before 12 years old) or late menopause (after 50 years old) is also a risk factor of developing endometrial cancer [25]. However, starting menstrual periods early (before 12 years old) is less a risk factor for women with early menopause. Likewise, late menopause may not lead to a higher risk in women whose menstrual periods began later in their early life. In our current study, our data shows that only $3.3 \%$ of all patients had early menarche (younger than 12 years old). The menstrual period time for these patients was 37 years, which suggests there was no late menopause as the natural menopausal age of Chinese women is 49.5 years old [26]. In our current study, our data shows that the median age at menopause of patients who diagnosed after menopause was 50 years old.

China instituted a "one child policy" at the end of the 1970s. In practice, this policy was implemented and had more effect in the urban rather than rural areas. In this study the median age of patients at diagnosis was 55 years old (ranging from 21- 85). Majority of the patients $(76 \%)$ who had more than two live births were over 55 years old and were not restricted by this policy.

There are some limitations to this study. Data were obtained from two Obstetrics \& Gynaecology university teaching hospitals in two different regions in China. This limited the sample size, both in terms of numbers and geographical areas covered. The association between parity and time of developing endometrial cancer may vary among regions and economic levels in China.

In conclusion, our study suggests that Chinese women were younger at presentation of endometrial cancer and parity is negatively correlated with the age of patients at diagnosis of endometrial cancer regardless of whether the patients were diagnosed before or after menopause. Our data also suggests that parity may not be associated with the incidence of subtype of endometrial cancer.

\section{Acknowledgements}

This study was supported by the Chinese National Nature Sciences Foundation (grant number 81100437 to M Zhao). We would like to thank Dr. Greg Gamble from the University of Auckland for statistical analysis help.

\section{Competing Interests}

The authors have declared that no competing interest exists.

\section{References}

1. Stewart BW and Wild C: World cancer report. Lyon, France. 2014.

2. Setiawan VW, Pike MC, Karageorgi S, et al: Age at last birth in relation to risk of endometrial cancer: pooled analysis in the epidemiology of endometrial cancer consortium. Am J Epidemiol, 2012. 176(4): 269-78.

3. Nichols HB, Trentham-Dietz A, Hampton JM, Titus-Ernstoff L, Egan KM, Willett WC and Newcomb PA: From menarche to menopause: trends among US Women born from 1912 to 1969. Am J Epidemiol, 2006. 164(10): 1003-11.

4. Karageorgi S, Hankinson SE, Kraft P and De Vivo I: Reproductive factors and postmenopausal hormone use in relation to endometrial cancer risk in the Nurses' Health Study cohort 1976-2004. Int J Cancer, 2010. 126(1): 208-16.

5. Kaaks R, Lukanova A, and Kurzer MS: Obesity, endogenous hormones, and endometrial cancer risk: a synthetic review. Cancer Epidemiol Biomarkers Prev, 2002. 11(12): 1531-43.

6. Jamison PM, Noone AM, Ries LA, Lee NC, and Edwards BK: Trends in endometrial cancer incidence by race and histology with a correction for the prevalence of hysterectomy, SEER 1992 to 2008. Cancer Epidemiol Biomarkers Prev, 2013. 22(2): 233-41.

7. Dossus L., Allen N., Kaaks R., et al. Reproductive risk factors and endometrial cancer: the European Prospective Investigation into Cancer and Nutrition. Int J Cancer, 2010. 127(2): 442-51.

8. Cook LS, et al. Endometrial cancer: Cancer epidemiology and prevention. New York: Oxford University Press. 2006.

9. Miller A.B., Barclay T.H., Choi N.W., Grace M.G., Wall C., Plante M., Howe G.R., Cinader B., and Davis F.G. A study of cancer, parity and age at first pregnancy. J Chronic Dis, 1980. 33(10): 595-605.

10. Cook L.S., Kmet L.M., Magliocco A.M., and Weiss N.S. Endometrial cancer survival among U.S. black and white women by birth cohort. Epidemiology, 2006. 17(4): 469-72.

11. Schonfeld S.J., Hartge P., Pfeiffer R.M., et al. An aggregated analysis of hormonal factors and endometrial cancer risk by parity. Cancer, 2013. 119(7): 1393-401.

12. Hoffman B., Schorge J., Schaffer J., et al. Gynecology: Endometrial Cancer 2nd ed. McGraw-Hill. 2012.

13. Schorge J.O and Williams J.W. Gynecology. New York: McGraw-Hill Medical. 2012.

14. Olson S.H., Atoria C.L., Cote M.L., Cook L.S., Rastogi R., Soslow R.A., Brown C.L., and Elkin E.B. The impact of race and comorbidity on survival in endometrial cancer. Cancer Epidemiol Biomarkers Prev, 2012. 21(5): 753-60.

15. Mahdi H., Schlick C.J., Kowk L.L., Moslemi-Kebria M., and Michener C. Endometrial cancer in Asian and American Indian/Alaskan Native women: tumor characteristics, treatment and outcome compared to non-Hispanic white women. Gynecol Oncol, 2014. 132(2): 443-9. 
16. Akhmedkhanov A., Zeleniuch-Jacquotte A., and Toniolo P. Role of exogenous and endogenous hormones in endometrial cancer: review of the evidence and research perspectives. Ann N Y Acad Sci, 2001. 943: 296-315.

17. Do K.A., Treloar S.A., Pandeya N., Purdie D., Green A.C., Heath A.C., and Martin N.G. Predictive factors of age at menopause in a large Australian twin study. Hum Biol, 1998. 70(6): 1073-91.

18. Setiawan V.W., Pike M.C., Kolonel L.N., Nomura A.M., Goodman M.T., and Henderson B.E. Racial/ethnic differences in endometrial cancer risk: the multiethnic cohort study. Am J Epidemiol, 2007. 165(3): 262-70.

19. Evans T., Sany O., Pearmain P., Ganesan R., Blann A., and Sundar S. Differential trends in the rising incidence of endometrial cancer by type: data from a UK population-based registry from 1994 to 2006. Br J Cancer, 2011. 104(9): 1505-10.

20. Lentz G.M. Comprehensive gynecology. 6th ed. Philadelphia: Mosby Elsevier. 2013.

21. Kim J.J. and Chapman-Davis E. Role of progesterone in endometrial cancer. Semin Reprod Med, 2010. 28(1): 81-90.

22. Key T.J and Pike M.C. The dose-effect relationship between 'unopposed' oestrogens and endometrial mitotic rate: its central role in explaining and predicting endometrial cancer risk. Br J Cancer, 1988. 57(2): 205-12.

23. Henderson B.E. and Feigelson H.S. Hormonal carcinogenesis. Carcinogenesis, 2000. 21(3): 427-33

24. Setiawan V.W., Yang H.P., Pike M.C., et al. Type I and II endometrial cancers: have they different risk factors? J Clin Oncol, 2013. 31(20): 2607-18.

25. Vale C.L., Tierney J., Bull S.J., and Symonds P.R. Chemotherapy for advanced, recurrent or metastatic endometrial carcinoma. Cochrane Database Syst Rev, 2012. 8: CD003915.

26. Yang D., Haines C.J., Pan P., Zhang Q., Sun Y., Hong S., Tian F., Bai B., Peng X., Chen W., Yang X., Chen Y., Feng H., Zhao S., Lei H., Jiang Z., Ma X., and Liao W. Menopausal symptoms in mid-life women in southern China. Climacteric, 2008. 11(4): 329-36. 